\title{
TCDD inhibits spontaneous differentiation in human embryonic stem cells
}

\author{
Elyse A. Bolterstein ${ }^{1, \#}$ and B. Lynn Allen-Hoffmann ${ }^{1,2,3, *}$ \\ ${ }^{1}$ Molecular and Environmental Toxicology Center, ${ }^{2}$ Department of Pathology and Laboratory Medicine, \\ ${ }^{3}$ Department of Surgery, University of Wisconsin-Madison, 53706, USA.
}

\begin{abstract}
Pluripotent human embryonic stem (ES) cells provide a consistent developmental model for studying contaminant-induced changes in human cell fate and early developmental processes. Thus far, no studies have investigated the effects of 2, 3, 7, 8-tetrachlorodibenzo-p-dioxin (TCDD) exposure on the growth and differentiation of human ES cells. Here we show that the aryl hydrocarbon receptor (AhR) pathway is functional in human ES cells based on the ability of TCDD to induce the AhR/Arnt-dependent transcription of CYP1A1. No changes in colony morphology were observed in undifferentiated human ES cells following 8 days of treatment with TCDD. However, when human ES cells were allowed to replicate and spontaneously differentiate for 15 days in the presence of TCDD, colonies exhibited less morphological differentiation compared to control human ES cell cultures. This observation was confirmed on the molecular level by lower expression of markers of meso- and endodermal differentiation and higher expression of the pluripotency marker, Oct4, in TCDDtreated cultures. Additionally, TCDD inhibited the
\end{abstract}

\footnotetext{
*Corresponding author: B. Lynn Allen-Hoffmann, 5605 MSC, 1300 University Ave., Madison, WI 53706, USA.

blallenh@wisc.edu

"Present address: Elyse A. Bolterstein, 352A BBH, 5500 N. St. Louis Ave., Chicago, IL 60625, USA.

E-Bolterstein@neiu.edu
}

fibroblastic morphology resulting from the epithelial to mesenchymal transition (EMT) of spontaneously differentiating ES cells, preserving an undifferentiated cellular phenotype. Furthermore, lower expression of EMT markers as well as a lower incidence of the mesenchymal marker, $\mathrm{N}$-cadherin, was observed around the edges of TCDD-treated cell colonies. These findings suggest that TCDD treatment inhibits human ES cell differentiation, potentially through the inhibition of an EMT-like process.

KEYWORDS: stem cells, differentiation, TCDD, epithelial to mesenchymal transition, AhR, Oct4

\section{INTRODUCTION}

A constant challenge confronting the field of developmental toxicology is the creation of in vitro models that accurately predict toxicity of environmental contaminants in humans. Historically, in vivo animal models have been used to investigate embryotoxicity of xenobiotic compounds. However, high costs associated with in vivo studies, coupled with disparities in species-specific toxicological responses, necessitated implementation of in vitro developmental assays that employ the use of human cell lines. In vitro studies using human cells are often performed using primary cell lines, which lose proliferative capability in culture, or using immortalized cell lines, which predominantly consist of an altered genetic profile [1]. Through their pluripotent nature and unlimited proliferative ability, human embryonic stem (ES) cells have the potential to provide a promising solution to the limitations of primary human cells. 
By definition, pluripotent human ES cells have the ability to differentiate into any of the three major germ layers: ectoderm (neural and epithelial cells), mesoderm (hematopoetic, skeletal, and muscle cells), or endoderm (hepatic and pancreatic cells). Pluripotency of a particular human ES cell line is determined both by the expression of genetic markers such as Oct4, as well as the generation of all three germ layers in human ES cell-initiated teratomas [2]. Human ES cell culture conditions can be manipulated to either maintain pluripotency or encourage differentiation that is either spontaneous or formulated to drive cells toward a specific lineage.

In recent years, the need to develop animal-free toxicological screening strategies for programs such as REACH (Registration, Evaluation, Authorization and Restriction of Chemicals) in the European Union has influenced the creation of human ES cell-based tests to identify developmental toxins (reviewed in [3]). Most toxicological screening strategies employ either directed or spontaneous differentiation culture methods, although limited studies on pluripotent human ES cells have been published (reviewed in $[4,5])$. Directed differentiation of human ES cells and induced pluripotent stem (iPS) cells has been accomplished through supplementation of culture media with specific growth factor combinations to encourage differentiation into a specific lineage. Because of the importance of heart and liver tissue in traditional toxicity screening, testing protocols for human ES cell-derived cadiomyocytes and hepatocytes are currently the most developed (reviewed in [3]). However, these strategies do not consistently result in homogeneous populations of the desired cell type, and full characterization of human ES-derived cell types must be completed before these cells can be used reliably for testing purposes in place of primary cells isolated from human tissues.

Other studies that use ES cells as a toxicological model employ an undirected differentiation strategy to produce various cell types and tissue-like masses. The embryonic stem cell test (EST), a teratogen screening assay for both mouse and human ES cells which utilizes cardiomyocyte-like embryoid bodies (EBs), has successfully predicted embryotoxicity of compounds such as methylmercury, retinoic acid, and arsenic (reviewed in [4, 5]). Although the EST is highly amenable to highthroughput screening, its simple endpoints, such as cytotoxicity, do not provide sufficient information for detailed mechanistic studies of developmental toxicity.

Exposure to 2, 3, 7, 8-tetrachlorodibenzo-p-dioxin (TCDD) has been shown to induce various deleterious effects on metabolism, cellular differentiation and organ function, usually mediated through the aryl hydrocarbon receptor (AhR) pathway (reviewed in $[6,7])$. Though carcinogenic and teratogenic in rodents, the only documented human toxic response to TCDD is chloracne, in which hyperkeratinization of skin cells results in the development of non-inflammatory comodones. Recent evidence suggests that this pathology results from TCDD-induced damage to a region adjacent to the hair follicle known as the bulge region, which is a known location of epithelial stem cells [8].

Though effects of TCDD on cellular differentiation have been shown to vary based on tissue type and species [7], the inhibitory effect of TCDD on the process of epithelial to mesenchymal transition (EMT) in rodent palatal development has been well described [9, 10]. Genital malformations such as cleft clitoris and incomplete vaginal openings, as well as delayed maturation of mammary and uterine tissue have also been described, further suggesting an inhibitory effect of TCDD on cellular differentiation and early developmental processes in rodent models [11-13].

The effects of TCDD exposure on growth and differentiation of human ES cells are unexplored. For this reason we investigated the impact of TCDD on human ES cells in an undifferentiated state as well as in conditions permissive of spontaneous differentiation. Here we show that in human ES cells cultured under conditions permissive for differentiation, TCDD treatment forestalled expression of cell differentiation markers with attenuation most prominently observed in mesodermal, endodermal and trophoblast developmental markers. Additionally, TCDDtreated cells exhibited lower expression and altered localization of EMT markers. Our studies on human ES cells have extended the early seminal findings in rodent cleft palate models to 
early human development. The findings suggest that TCDD exposure promotes human ES cell pluripotency and inhibits cellular differentiation of human ES cells.

\section{MATERIALS AND METHODS}

\section{Maintenance cell culture}

Human ES cells from the $\mathrm{H} 1$ and $\mathrm{H} 9$ cell lines (WiCell, Madison, WI) were cultured on a feeder layer of mitomycin-C-inactivated 3T3 mouse fibroblasts, which has been shown to support pluripotent human ES cell cultivation similar to human ES cells cultured on feeder layers generated from mouse embryonic fibroblasts (MEFs) [14]. Cells were maintained at $37{ }^{\circ} \mathrm{C}$ in a humidified chamber with $5 \% \mathrm{CO}_{2}$. Growth medium containing Dulbecco's modified Eagle's medium (DMEM)/F12 medium (Invitrogen, Carlsbad, CA) supplemented with $20 \%$ knockout serum replacement, $1 \%$ nonessential amino acids, $1 \mathrm{mM}$ L-glutamine, $0.1 \mathrm{mM} \quad \beta$-mercaptoethanol and $4 \mathrm{ng} / \mathrm{ml}$ basic fibroblast growth factor (bFGF) was changed daily. Undifferentiated stock cultures were maintained by mechanically passaging cells every 7-10 days. All experiments were performed using H9 cells except RNA analysis of suspension cultures, which used independent cultures of both H9 cells and H1 cells.

\section{Experimental cell culture}

Semi-confluent human ES cells (approximately $5 \times 10^{5}$ cells) were mechanically dissociated in human ES medium divided evenly and passaged onto 3T3 feeder cells on 6-well culture plates. Cells were given medium containing either $10 \mathrm{nM}$ TCDD (Wellington Laboratories, Guelph, Ontario, Canada) in 0.1\% DMSO (total volume) or $0.1 \%$ DMSO alone beginning on day 2 of culture (day 0 referring to date of cell passage). For the majority of experiments described, undifferentiated cells were harvested on days 5 and 8 of culture, while differentiating cells were harvested on day 15 of culture. Morphology of human ES cell colonies was evaluated prior to cell harvest using standard phase-contrast microscopy.

\section{Analysis of cell growth characteristics}

To determine colony growth characteristics of undifferentiated cells displayed in Figure 2C, cells were grown as described above on $35 \mathrm{~mm}$ gridded culture plates (Corning Inc., Corning, NY). Images of each of the 36 centrally located grid squares were captured at days 5 and 8 of culture using an Olympus IX-71 inverted fluorescent microscope equipped with FITC (490 $\pm 20 \mathrm{~nm})$, Texas Red $(490 \pm 20 \mathrm{~nm})$, and Hoechst $(360 \pm 20 \mathrm{~nm})$ band pass filters, an Olympus DP70 digital camera and DP Controller software (Olympus, Center Valley, PA). Images were analyzed with ImageJ software (available at http://rsb.info.nih.gov/ij; developed by Wayne Rasband, National Institutes of Health, Bethesda, MD) to determine colony count, size, and area.

Differentiation status was scored on day 8 (Figure 2C) or day 15 (Figure 3B) using a scoring system ranging in value from 1 (undifferentiated colonies) to 6 (highly differentiated colonies). An example of this scoring rubric is given in Figure 2A. Statistics were determined by combining differentiation scores from each grid (36 per culture) from 5 independent experiments, using GraphPad Prism 5 (GraphPad Software Inc., La Jolla, CA) to determine significance via one-way ANOVA and Bonferroni's multiple comparison post-test. A 95\% confidence interval was used. Data is presented as mean and SEM.

To determine colony growth of spontaneously differentiating human ES cells, cells were grown for 15 days as previously described on 6-well culture plates. On day 3 of culture, 12 colonies were selected at random in both TCDD- and DMSO-treated cultures. Images were captured of each of these selected colonies every 3 days of culture using the imaging system previously described. Colony diameter was measured at each timepoint using ImageJ software.

\section{Suspension culture}

Human ES cells were cultured to 40-50\% confluency (10-12 days) on 3T3 feeder cells as described above. Cells were harvested by trypsinization and transferred to a $50 \mathrm{~mL}$ conical tube containing medium with $1.68 \%$ methocellulose to create a single-cell suspension of approximately $10^{5}$ cells $/ \mathrm{mL}$. Cultures were maintained at $37^{\circ} \mathrm{C}$ until harvest. Aliquots of the suspension were collected at 2-, 6-, 12- and 24-hour timepoints, rinsed with DMEM/F12 and resuspended in Trizol ${ }^{\mathrm{TM}}$ reagent (Invitrogen, Carlsbad, CA) for RNA analysis. 


\section{Reverse transcriptase polymerase chain reaction (RT-PCR)}

For 5- and 8-day cultures, total RNA was obtained from the human ES cells by the addition of $1 \mathrm{ml}$ Trizol $^{\mathrm{TM}}$ reagent per well, following the removal of 3T3 feeder cells by mechanical dislodging with a $5 \mathrm{~mL}$ glass pipette. Following 15 days of culture, it was not possible to separate human ES cells from feeder cells by mechanical means and therefore both cell lines were collected in Trizol $^{\mathrm{TM}}$. Total RNA was isolated according to manufacturer's directions. cDNA was processed from total isolated RNA using M-MLV Reverse Transcriptase reagents (Promega, Madison, WI). cDNA was diluted to a concentration of 0.02 $\mu \mathrm{g} / \mu \mathrm{L}$ and added to 5 Prime Perfect Taq DNA Polymerase and associated buffers (5 PRIME Inc., Gaithersburg, MD), $10 \mathrm{nM}$ deoxyribonucleotide triphosphates (dNTPs), and oligo primer sets (Integrated DNA Technologies, Coralville, IA) in $25 \mu \mathrm{L}$ final reaction volumes. Human-specific primer sets within genes encoding the following proteins were designed to eliminate confounding signals from feeder cells: Cyclophilin A: FCAAGGTCCCAAAGACAGCAGAA, R- CCACC CTGACACATAAACCCTG; Oct4: F- TGGGGG TTCTATTTGGGAAGG, R- GTTCGCTTTCTCT TTCGGGC; Nestin: F- AGCGTTGGAACAGAG GTTG, R- GCTGAGGGAAGTCTTGGAG; BMP4: F- GTTTGTTCAAGATTGGCTGTC, R- AAGA TCCCGCATGTAGTCC; Vimentin: F- CCAGCC GCAGCCTCTACG, R- GCGAGAAGTCCACC GAGTCC; AFP: F- CTTTGGGCTGCTCGCTAT GA, R- TGGCTTGGAAAGTTCGGGTC; FoxA1: F- TCATGTTGCCGCTCGTAGTC, R- AGGAA CTGTGAAGATGGAAGGG; Hand1: F- TGCCT GAGAAAGAGAACCAG, R- TGCCTGAGAAA GAGAACCAG; Snail: F- ACCGCCTCGCTGCC AATG, R- AGCCTTTCCCACTGTCCTCATC; Slug: F- CTCCCGCCGGACCGTTATC, R- AG CTGAGGATCTCTGGTTGTGG; MMP-2: F- CG CCCATCATCAAGTTCC, R- TGTCCTTCAGC ACAAACAGG; MMP-9: F- CAGTCCACCCTT GTGCTCTT, R- CTATCCAGCTCACCGGTCTC; E-cadherin: F- CTCCCGCCGGACCGTTATC, R- AGCTGAGGATCTCTGGTTGTGG; TIMP1: F- ACTTCCACAGGTCCCACAAC, R- TTTGC AGGGGATGGATAAAC; Bax: F- CCAGAGGC GGGGTTTCATCC, R- TCCACGGCGGCAATC
ATCC; Bcl2-L1: F- GACTGAATCGGAGATG GAGACC, R- TCTACGCTTTCCACGCACAG; Cyclin D2: F- ATCGCAACTGGAAGTGTGG, R- GAGCACCGCCTCAATCTG; Wnt1: F- AG CTGAGGATCTCTGGTTGTGG, R- AGGAGGC AGGACAGGAAGG; Wnt3: F- CTTCCACTGG TGCTGCTACG, R- CTTGCTGTCTGACGCTG AGG.

Thermocycler reactions were run for 32 cycles at an annealing temperature of $57^{\circ} \mathrm{C}$. Reactions were then subjected to electrophoresis using a $2 \%$ agarose gel.

\section{Quantitative polymerase chain reaction (qPCR)}

Total RNA was obtained and converted to cDNA as described above. Quantitative polymerase chain reaction (qPCR) was performed using the following Taqman ${ }^{\circledR}$ Gene Expression Assays (Applied Biosystems, Foster City, CA): Cyclophilin: NM_021130.3, Arnt: Hs00231048_m1, AhR: Hs00169233_m1, CYP1A1: Hs00153120_m1. Assays were executed in triplicate according to manufacturer's directions using 50 ng cDNA per $20 \mu \mathrm{L}$ reaction. The reactions were processed by the Chromo4 Real-Time PCR Detection System (Bio-Rad Laboratories, Hercules, CA) following the manufacturer's protocol. $\mathrm{C}(\mathrm{t})$ values were normalized to Cyclophilin A and analyzed by the GenEx Macro iQ5 Conversion Program (Bio-Rad Laboratories, Hercules, CA) and GraphPad Prism 5. Statistics were determined by unpaired t-test, using $95 \%$ confidence intervals. Data is presented as mean and SEM.

\section{Immunoblot analysis}

Whole cell lysates were collected by manual detachment of human ES cells from 6-well culture plates into $300 \mu \mathrm{L}$ Cytobuster (Novagen, San Diego, CA) containing protease inhibitor cocktail set III (Calbiochem, San Diego, CA). Cell lysates were passed through a 23 gauge needle to disaggregate cell clumps and centrifuged at $12,000 \mathrm{x} \mathrm{g}$ for $15 \mathrm{~min}$ at $4{ }^{\circ} \mathrm{C}$. Total protein concentration was determined by bicinchoninic acid (BCA) assay (Pierce, Rockford, IL). $20 \mu \mathrm{g}$ protein for each experimental condition was separated on a NuPAGE 4-12\% Bis-Tris gel (Invitrogen, Carlsbad, CA) as per manufacturer's protocol. Proteins were then electrophoretically 
transferred onto a $0.2 \mu \mathrm{m}$ polyvinylidene fluoride (PVDF) membrane (Millipore, Billerica, MA) using an XCell blot module (Invitrogen, Carlsbad, CA). Following blocking in 2\% Enhanced Chemiluminescence (ECL) Advance blocking reagent (Amersham Biosciences, Pittsburgh, PA), blots were probed using monoclonal antibody against Oct3/4 (sc-5279; Santa Cruz Biotechnology, Inc., Santa Cruz, CA), N- or E-cadherin (BD Biosciences, San Jose, CA), or $\alpha$-tubulin as a loading control, diluted 1:1000 in blocking reagent. Blots were then incubated with a horseradish peroxidase-coupled goat anti-rabbit IgG antibody diluted 1:50,000 in blocking reagent (Promega, Madison, WI). The antibody hybridized membrane was then developed with an ECL system (Amersham Biosciences, Pittsburgh, PA) and visualized using a Kodak Image Station 2000R (Kodak, New Haven, CT).

\section{Indirect immunofluorescence analysis (IIF)}

Cells were fixed in situ in a solution of $1 \%$ paraformadehyde in phosphate buffered saline (PBS) for 1 hour and subsequently stored in PBS. Following a blocking step using 3\% normal goat serum (NGS; Sigma, St. Louis, MO), sections were incubated with primary antibodies anti-Ecadherin (R \& D Systems, Minneapolis, MN; diluted 1:50), anti-N-cadherin (BD Biosciences, San Jose, CA; diluted 1:50), or anti-Oct3/4 (Santa Cruz Biotechnology, Inc., Santa Cruz, CA; diluted 1:200) in NGS, followed by incubation with secondary antibody IgG-Alexa Fluor 488 or IgGAlexa Fluor 594 (Molecular Probes, Eugene, OR) diluted 1:1000 in NGS. Cultures were counterstained with $5 \mu \mathrm{g} / \mathrm{mL}$ Hoechst 33258. Samples were reviewed using the imaging system described previously.

\section{RESULTS}

\section{AhR and Arnt are present and functional in human ES cells}

To determine if AhR pathway members were present and functional in undifferentiated human ES cells, cultures were analyzed after 5 and 8 days of growth for mRNA expression. Transcripts from the aryl hydrocarbon receptor and aryl hydrocarbon receptor nuclear translocator loci (AHR and ARNT, respectively) were expressed in monolayer cultures at culture day 5 (Figure 1A). In 8-day cultures, both $A H R$ and $A R N T$ levels increased relative to the 5-day cultures, suggesting that $A H R$ and $A R N T$ levels may increase with culture time. To determine if aryl hydrocarbon receptor and aryl hydrocarbon receptor nuclear translocator proteins (AhR and Arnt, respectively) were functional in undifferentiated human ES cells, transcription of a well-characterized AhR-responsive gene, cytochrome P450 1A1 (CYP1A1), was monitored. AhR signaling was induced by exogenous and endogenous strategies. When TCDD treatment was used to exogenously activate the AhR pathway, a 40 to 60 -fold increase in CYP1A1 expression was observed in TCDD-treated cells compared to timematched controls at both timepoints (Figure 1B). These findings indicate that $\mathrm{AhR}$ and Arnt are present in pluripotent human ES cells and can be activated by TCDD.

The AhR pathway can also be activated endogenously by disrupting cell-cell adhesion by single-cell suspension culture [15]. Undifferentiated human ES cells were suspended in semi-solid methocellulose media and analyzed at 2, 6, 12 and 24 hours. Quantitative PCR analysis showed upregulation of CYP1A1 at 2, 6 and 12 hours, which returned to baseline at 24 hours (Figure 1C). Suspension analysis of the H1 cell line produced similar results (data not shown).

\section{TCDD does not alter cellular morphology in human ES cells}

Despite the fact that TCDD is known to influence cellular differentiation in early mammalian embryogenesis, no studies have been published investigating TCDD effects in human ES cells. Therefore, undifferentiated human ES cells were visually monitored after 8 days of growth to determine TCDD-induced changes in growth and differentiation properties. Based on previous work in our laboratory showing that TCDD enhanced keratinocyte terminal differentiation and stratification, we expected to observe higher levels of differentiated human ES cells following TCDD exposure [16]. Colony morphology was scored on a scale from 1 to 6 to represent the extent of spontaneous differentiation visible (Figure 2A). However, phase-contrast microscopy revealed no apparent differences in cellular or colony morphology of TCDD-treated undifferentiated 

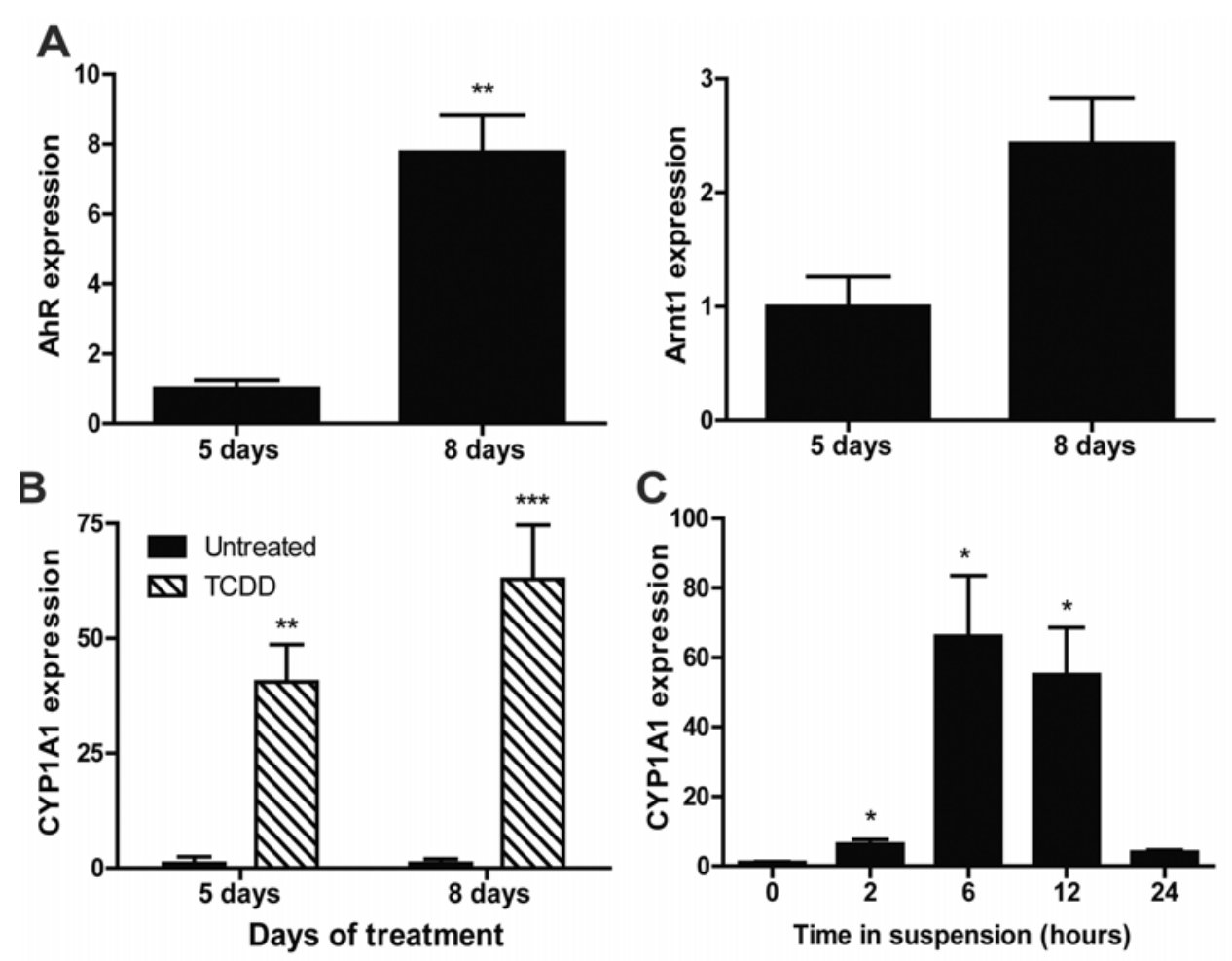

Figure 1. The AhR pathway is functional in human ES cells. qPCR was used to demonstrate the presence of AhR and Arnt transcripts in undifferentiated human ES H9 cells grown in monolayer culture for 5 and 8 days (A). AhR pathway functionality was demonstrated through the upregulation of CYP1A1 following either TCDD exposure (B) or suspension in medium containing $1.68 \%$ methylcellulose $(\mathbf{C})$. $C(t)$ values were obtained in triplicate, normalized to Cyclophilin A and compared to DMSO-treated controls when available (DMSO controls contained undetectable CYP1A1 transcript and an untreated control was substituted for comparison to TCDD-treated cells). Each graph presents a representative outcome generated from at least three independent experimental repeats. ${ }^{*} \mathrm{p}<0.05, * * \mathrm{p}<0.01,{ }^{* * *} \mathrm{p}<0.001$.

human ES cells compared to vehicle controls (Figure 2B). In addition, quantification of colony number, diameter, and area, confirmed no significant differences between TCDD-treated human ES cell colonies and DMSO controls (Figure 2C).

\section{TCDD inhibits cellular morphological differentiation in 15-day human ES cells}

The morphology of undifferentiated human ES cells, which exhibit characteristics of a pluripotent cell population, was unaffected by TCDD. However, TCDD has been shown to either increase or decrease differentiation in a tissue- and cell-type specific manner [7]. Therefore, we created a culture environment permissive for spontaneous differentiation, enabling the formation of differentiated cell types that may be more susceptible to TCDD. In order to create a differentiation- permissive culture environment, we increased the culture time to 15 days, at which time spontaneous differentiation could be reliably observed and quantified. At day 15, cell colonies were assessed for morphological changes by standard phasecontrast microscopy and harvested for total RNA. Expression of $A H R$ and $A R N T$ was confirmed in 15-day human ES cells by qPCR analysis and functionality of the AhR-ARNT transcriptional complex was demonstrated through CYP1A1 induction (data not shown).

As expected, morphological properties indicative of spontaneous differentiation were observed in control human ES cultures. The periphery of the differentiating human ES cell colonies in the DMSO controls had become populated by fusiform, less compact cells reminiscent of fibroblast morphology (Figure 3A). Additionally, a high degree 
A
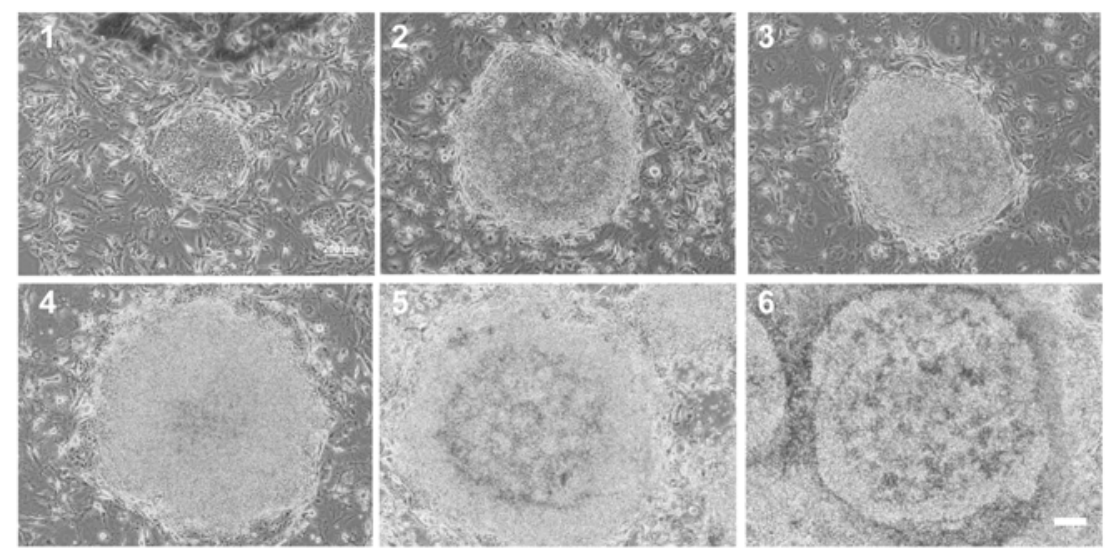

B
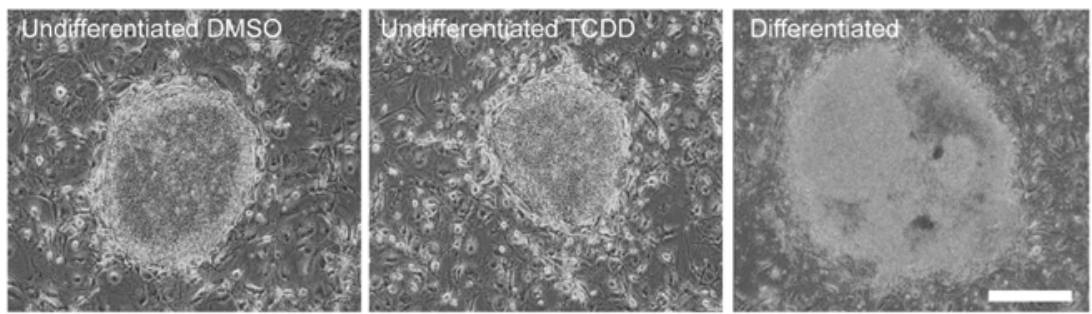

C

\begin{tabular}{|l|r|r|}
\cline { 2 - 3 } \multicolumn{1}{c|}{} & \multicolumn{1}{c|}{ DMSO } & \multicolumn{1}{c|}{ TCDD } \\
\hline Mean colony number & $15.7 \pm 1.5$ & $13 \pm 1.5$ \\
Mean colony diameter $(\mathrm{mm})$ & $1.2 \pm 0.12$ & $1.7 \pm 0.48$ \\
Total colony area $\left(\mathrm{mm}^{2}\right)$ & $54.8 \pm 21.2$ & $45.0 \pm 13.5$ \\
Mean differentiation score & $2.9 \pm 0.09$ & $2.7 \pm 0.26$ \\
\hline
\end{tabular}

Figure 2. TCDD treatment does not alter the colony morphology of undifferentiated human ES cells. To monitor the impact of TCDD, a scale was developed to characterize the extent of morphological differentiation in human ES cell colonies (A). Colonies were given a score between 1 (undifferentiated) and 6 (highly differentiated) as follows: 1 - circular shape, well-defined borders, tightly packed cells; 2 - mostly circular, borders becoming irregular, $<50 \%$ phase-dense material accumulation in colony center; 3 - mostly circular, evidence of some cell migration ( $<10$ cells outside of colony borders), borders irregular, $>50 \%$ phase-dense material accumulation in colony center; 4 - evidence of cell migration ( $\geq 10$ cells outside of colony borders), borders irregular, colony center covered by single-layer phase-dense material (no brown spots); 5 - evidence of cell migration ( $\geq 10$ cells outside of colony borders), borders irregular, colony center covered by multi-layer phase-dense material (dark in areas in center only); 6 - evidence of cell migration ( $\geq 10$ cells outside of colony borders), borders irregular, colony center and borders covered by multi-layer phase-dense material (dark in areas). A representative colony of each score is shown. Untreated cells grown 8 days in culture had an average score of approximately 3.0 whereas 15-day spontaneously differentiating cultures generally scored 4.5-6.0. Bar $=200 \mu \mathrm{m}$. (B) Two day cultures of undifferentiated ES cells were treated with $0.1 \%$ DMSO vehicle or TCDD for 6 days and examined for colony morphology using phase contrast microscopy. Cultures retained a mostly undifferentiated appearance (small cell size, high nucleus to cytoplasm ratio, and even colony borders). No differences in cell or colony characteristics were observed between vehicle and TCDD treatment during this time period. After 15 days in culture, untreated control human ES cell colonies demonstrated a greater abundance of mesenchymal-like cells and a higher degree of internal differentiation (right). Bar $=500 \mu \mathrm{m}$. (C) Human ES H9 cells were plated in equal densities on gridded $35 \mathrm{~mm}$ cell culture plates and treated with TCDD or DMSO vehicle beginning at day 2. On culture day 8, phase-contrast photographs were captured for each of the 36 grid squares $(3.5 \times 3.5 \mathrm{~mm})$ for a total analyzable culture area of $440 \mathrm{~mm}^{2}$. Images were analyzed visually for differentiation status, and colony measurements were obtained using ImageJ software. Values represent the means \pm SEM of three separate experiments. No significant differences were detected between treatments for any of these parameters, as analyzed using one-way ANOVA. 
of phase-dense material was observed inside the colonies, which is indicative of cellular differentiation. In contrast, TCDD-treated colonies retained a morphology characteristic of undifferentiated human ES cells including the presence of small, round, compact cells located in the colony's center, with fewer fibroblast-like cells at the colony's periphery. TCDD-treated colonies exhibited less phase-dense internal material and possessed a well-spread morphology that occupied more surface area. We quantified the extent of colony morphological differentiation using a differentiation scoring rubric (see Figure 2A). TCDD-treated cells exhibited significantly lower differentiation scores compared to DMSO controls (Figure 3B), suggesting that TCDD forestalls cell differentiation under culture conditions permissive for spontaneous differentiation.

\section{TCDD treatment alters expression of} pluripotency and differentiation markers in a population of differentiating human ES cells

To complement our finding that TCDD treatment forestalled morphological human ES cell differentiation, we assessed expression of the pluripotency marker Oct4 in a population of differentiating human ES cells. An inverse relationship between Oct4 levels and cellular differentiation has been well documented [2]. Although a decrease in Oct4 expression in human ES cells was expected for cells cultured under conditions permissive for differentiation, at day 15 , no differences in Oct4 transcript levels were observed when comparing DMSO-treated control and TCDD-treated human ES cells (Figure 4A). However, protein analysis by immunoblot and indirect immunofluorescence (IIF) demonstrated that relative to the DMSO control, TCDD-treated cells had higher levels of Oct4 expression (Figure 4B and C). Higher levels of Oct4 in differentiated cells may indicate that TCDD forestalls the ability of human ES cells to spontaneously differentiate in response to culture confluence, thus enabling them to maintain a pluripotent state.

Spontaneous differentiation of human ES cells induced by culture confluence results in a heterogeneous population of cells derived from
A
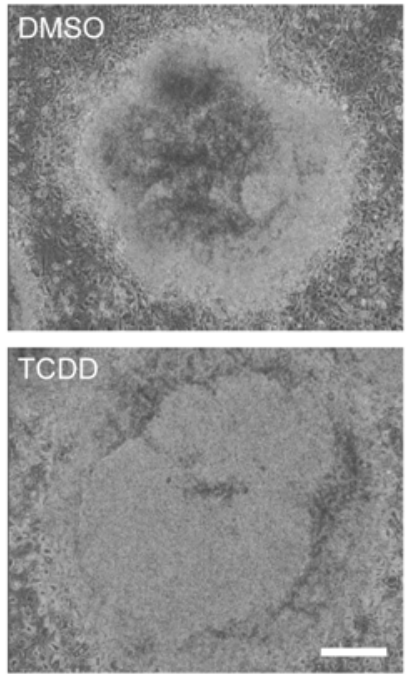

B

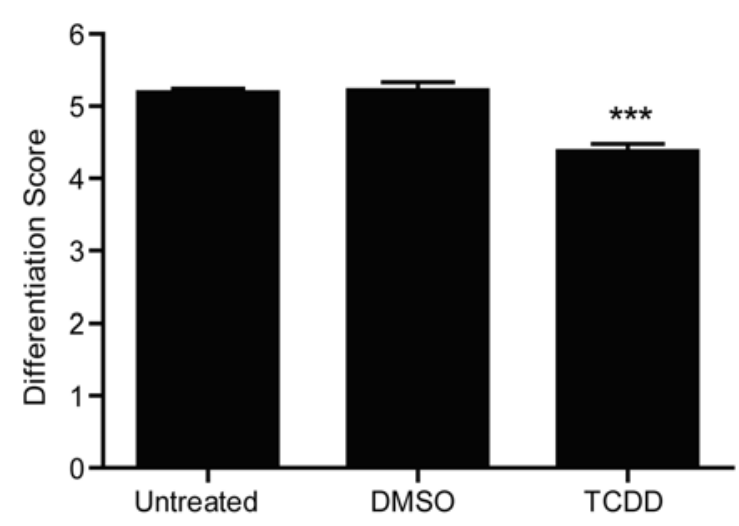

Figure 3. TCDD treatment retains undifferentiated morphology in spontaneously differentiating human ES cells. Colonies were visualized using phase contrast microscopy following 13 days of TCDD treatment (15 days total culture time). DMSO colonies demonstrated a greater abundance of mesenchymal-like cells and a higher degree of internal differentiation, while TCDD-treated colonies retained a more undifferentiated-like morphology as demonstrated by a round shape with clearly-defined borders and tightly packed cells with a large nucleus to cytoplasm ratio (A). Bar $=500 \mu \mathrm{m}$. These observations were further quantified using a differentiation scale where 1 represented an undifferentiated colony and a score of 6 indicated a high degree of differentiation (B) (see Figure 2A for morphology scale). Data is presented as means and SEM. ${ }^{* * *} \mathrm{p}<0.001$. 
A

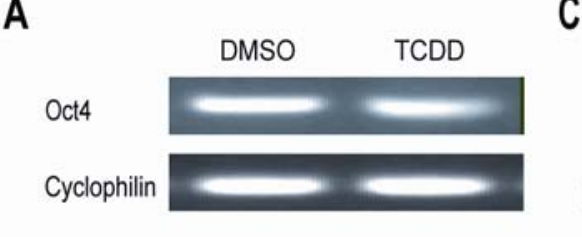

B

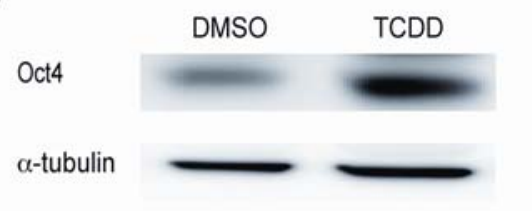

C

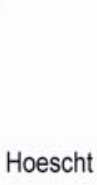

Oct4
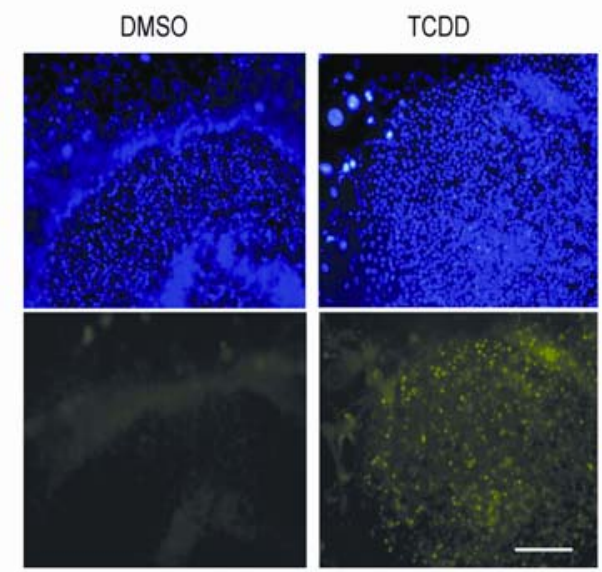

Figure 4. TCDD treatment alters expression of Oct4 in spontaneously differentiated human ES cells. Human ES cultures were treated with $10 \mathrm{nM}$ TCDD or DMSO for 13 days beginning at day 2 in culture (15 days total culture time). Transcript expression of the pluripotency marker Oct4 showed no differences following TCDD treatment using cyclophilin as a control for gene expression (A). However, Oct4 protein was observed in greater abundance following TCDD treatment as visualized though immunoblot analysis of whole cell lysates (B) and IIF (C), which may suggest a post-transcriptional mechanism of TCDD toxicity. Data shown are representative of three independent experimental repeats. Hoechst stain was used to identify nuclei. $\mathrm{Bar}=500 \mu \mathrm{m}$.

all three germ layers, ectoderm, mesoderm, and endoderm. We next determined the effect of TCDD treatment on expression of lineage markers in human ES cells using RT-PCR. Following 15 days of spontaneous differentiation, control cultures expressed genes representing ectodermal, mesodermal and endodermal lineages. However, parallel TCDD-treated cultures showed attenuated transcript expression for the ectodermal markers Nestin and NCAM1 (Figure 5). TCDD treatment also resulted in lower transcript levels for the mesodermal markers BMP4 and vimentin, as well as AFP and FoxA1, which are markers of primitive and definitive endoderm, respectively. Additionally, robust transcript accumulation of the trophoblast differentiation marker, Hand1, was observed in vehicle-treated control cultures, but not in TCDD-treated cultures. Taken together, these findings suggested that TCDD-treated cultures maintained a pattern of gene expression typical of pluripotent human ES cells [2].

\section{TCDD treatment inhibits an EMT-like process in spontaneously differentiating human ES cells}

To explore the effects of TCDD on the process of morphological differentiation in human ES cells we analyzed the expression and localization of

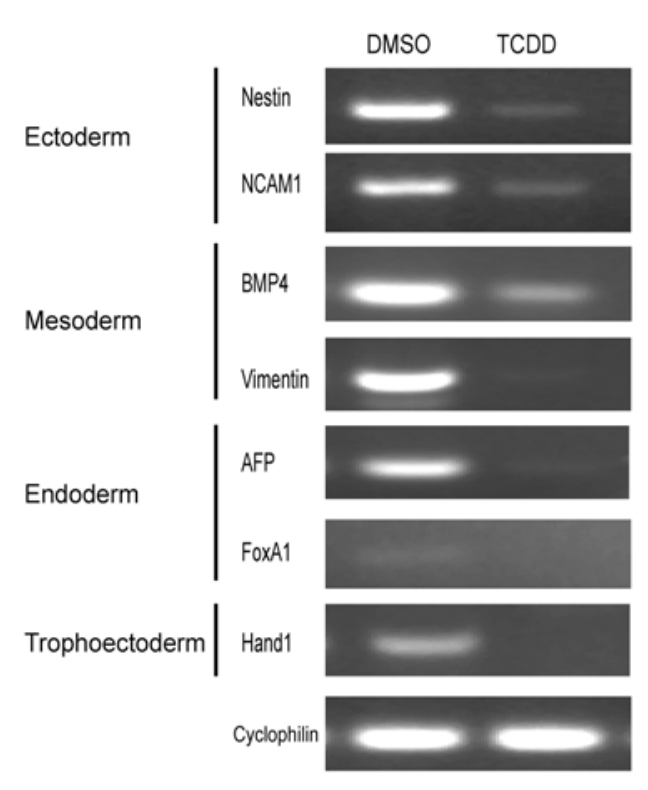

Figure 5. TCDD treatment alters expression of differentiation markers in spontaneously differentiating human ES cells. Human ES cultures were treated with either TCDD or DMSO for 13 days beginning at day 2 in culture (15 days total culture time). Expression of known markers of ecto-, meso- and endodermal differentiation were evaluated using RT-PCR, using cyclophilin as control for stable gene expression. Data shown are representative of three independent experimental repeats. 
E-cadherin and N-cadherin in 15-day cultures. The dynamic morphological changes and patterns of E-cadherin and $\mathrm{N}$-cadherin expression in differentiating human ES cells are tightly linked and have been attributed to the process of EMT [17-20], During EMT the cell adhesion protein E-cadherin becomes down-regulated and a different isoform, N-cadherin, is up-regulated resulting in changes in cell morphology and cell motility.
E-cadherin, an epithelial marker, was expressed in the center of cell colonies whereas the mesenchymal marker, $\mathrm{N}$-cadherin, was expressed in the fibroblastlike cells bordering the colony (Figure 6A). TCDD treatment resulted in reduced expression of the pro-EMT cell adhesion molecule $\mathrm{N}$-cadherin, but had no detectable effect on the expression or localization of anti-EMT protein E-cadherin (Figure 6B).
A

Phase

Hoescht

-cadherin/ $\mathrm{N}$-cadherin
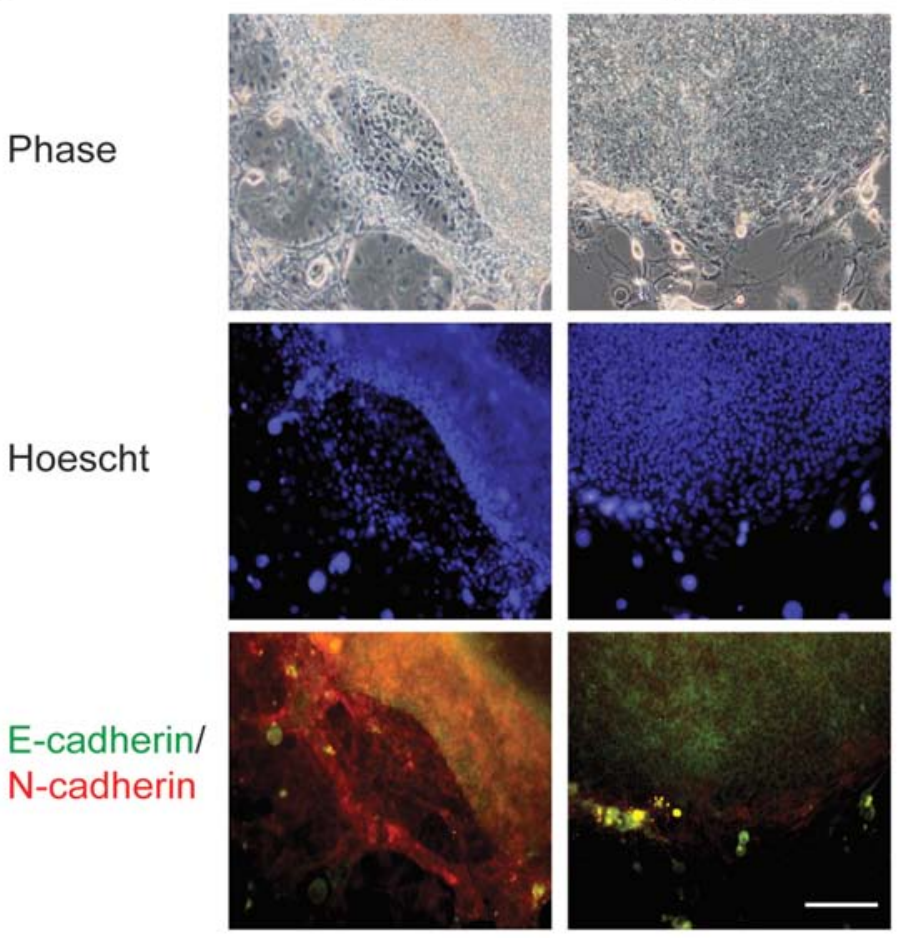

B

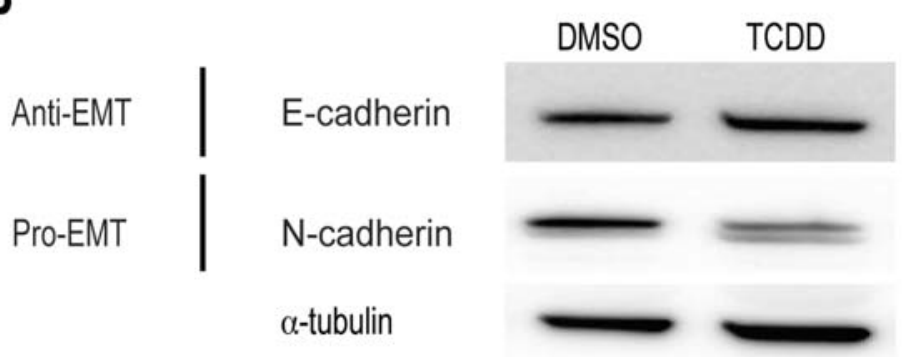

Figure 6. TCDD inhibits morphological signs of EMT in spontaneously differentiating human ES cells. Human ES cultures were treated with either TCDD or DMSO for 13 days beginning at day 2 in culture. Protein levels and localization of the EMT markers E-cadherin and N-cadherin were determined by IIF (A) and immunoblot (B). Nuclei were identified with Hoescht stain (blue). TCDD-treated cells exhibited less N-cadherin expression localized to the edges of colonies, indicating that TCDD inhibits EMT. Data shown are representative of three independent experimental repeats. Bar $=200 \mu \mathrm{m}$. 
To further investigate the effects of TCDD on human ES cell EMT, transcript levels for EMTrelated markers were assessed by RT-PCR. Control cultures of differentiating human ES cells expressed robust mRNA levels for the pro-EMT markers snail, slug, vimentin, and matrix metalloproteinases (MMP) 2 and 9. In contrast, TCDD treatment resulted in a dramatic reduction in mRNA levels for these same EMT markers (Figure 7). Notably, TCDD treatment blocked mRNA expression of the gene encoding slug, a transcription factor known to promote EMT through transcriptional regulation of E-cadherin. Unlike protein levels, E-cadherin transcript levels were lower in TCDD-treated cells compared to DMSO controls. The mRNA levels for tissue inhibitor of metalloproteinases (TIMP)-1 were not changed by TCDD treatment. TIMP-1 is a glycoprotein that directly inhibits most MMPs thereby preventing extracellular matrix (ECM) degradation and consequently EMT. Wnt1 and Wnt3 mRNA levels were also assessed because of their known roles in promoting EMT. Compared to controls, TCDD-treated cultures expressed no detectable Wnt1 mRNA and dramatically reduced levels of Wnt3. The level of $\beta$-catenin transcript was unaffected by TCDD treatment. These findings suggest that TCDD may interfere with both cellular differentiation and EMT in early stages of human ES differentiation.

\section{TCDD-induced effects on morphology and gene expression are not due to changes in cell proliferation or apoptosis}

Due to the TCDD-induced changes in mRNA expression for genes involved in differentiation and EMT, we determined if our observations reflected a direct effect of TCDD on human ES differentiation or was an indirect effect of cell proliferation or death. To assess the effect of TCDD on growth, the diameters of randomly selected human ES cell colonies were measured over the course of the 15-day culture period. Colony growth rate was calculated as an indirect measure of cell growth and proliferation. No difference in radial growth rate was observed between TCDD-treated colonies and untreated or vehicle controls (Figure 8A). It is important to note that these quantitative results were limited to two-dimensional radial growth; growth in the

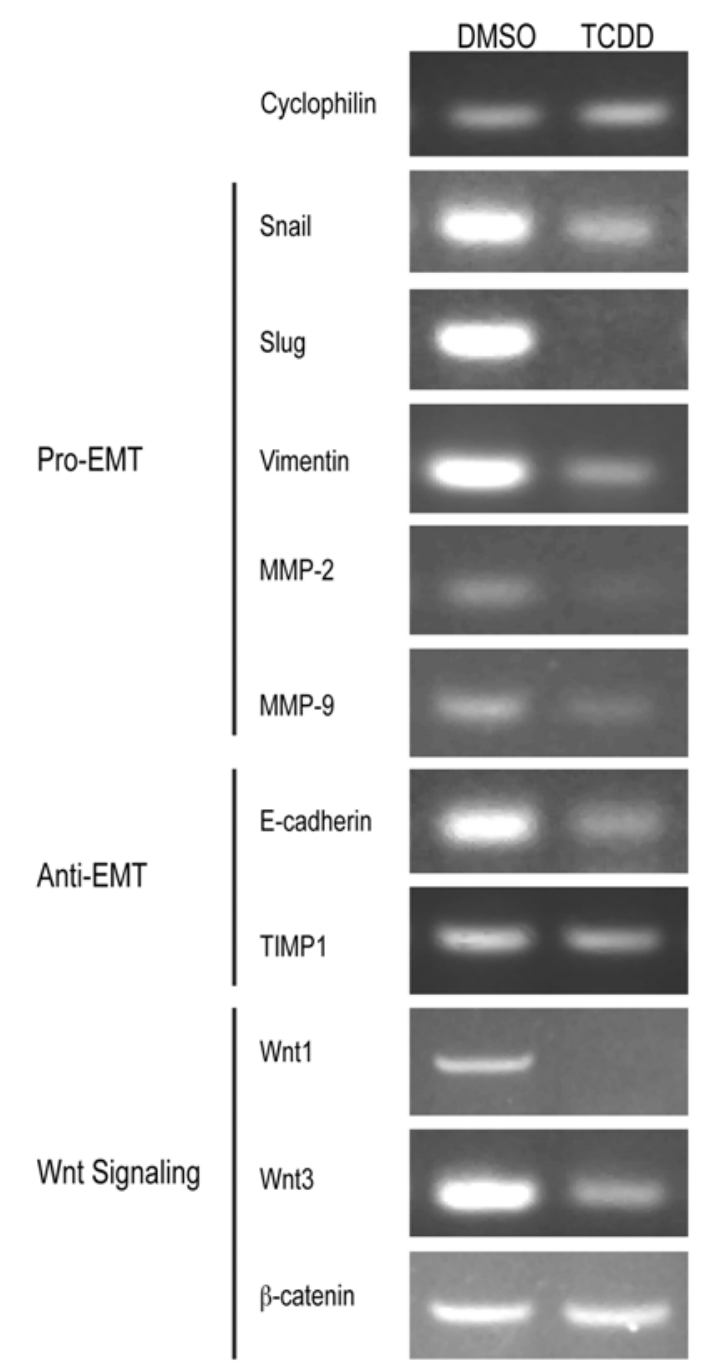

Figure 7. TCDD inhibits markers of EMT progression in spontaneously differentiating human ES. Human ES cultures were treated with either TCDD or DMSO for 13 days beginning at day 2 in culture. RT-PCR was used to determine expression of EMT related genes in TCDD-treated human ES cells compared to timematched untreated and DMSO-treated controls. TCDD exposure resulted in attenuation of pro-EMT markers, while anti-EMT markers displayed steady expression regardless of treatment, suggesting that TCDD inhibits EMT in differentiating human ES cells. Cyclophilin A was used as a control for stable gene expression. Data shown are representative of three independent experimental repeats.

vertical plane was not assessed. We also measured transcript levels of genes known to play key roles in cell cycle regulation and apoptosis. No consistent differences in mRNA levels for genes encoding 
A

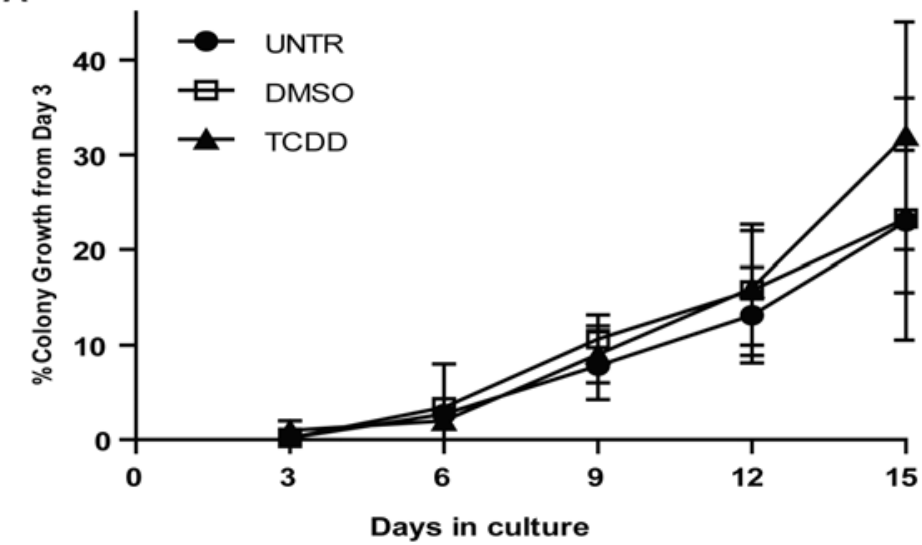

B

$\mathrm{BCl} 2-\mathrm{L} 1$

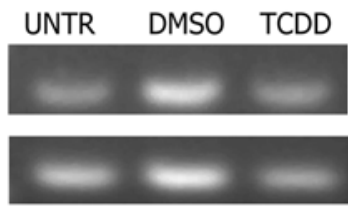

Cyclin D2

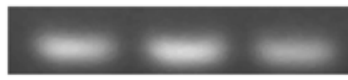

Cyclophilin

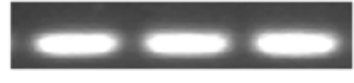

Figure 8. TCDD treatment does not alter expression of apoptosis and cell cycle regulation markers in spontaneously differentiated human ES cells. Monolayer human ES cell cultures were treated with 10 nM TCDD for 13 days beginning at day 2 in culture. Time-matched untreated or $0.1 \%$ DMSO-treated cultures served as controls. In order to compare growth rates between treatments, ImageJ software was used to measure colony diameter over the 15 day growth period starting at day 3 in culture. Displayed are colony sizes and standard deviations for each timepoint during one representative experiment (A). TCDD treatment did not change the steady state transcript levels of Bcl2-L1 (anti-apoptotic), Bax (pro-apoptotic) and Cyclin D2 (cell cycle regulation) (B).

Bax (pro-apoptosis), Bcl2-L1 (anti-apoptosis) and Cyclin D2 (cell cycle regulation) were observed among TCDD-treated, vehicle-treated controls, and untreated controls in human ES cells cultured under conditions that promote differentiation. These findings suggest that the TCDD-induced morphological and gene expression responses we have observed are likely due to alterations in cell fate and inhibition of human ES differentiation rather than altered cell proliferation or cell death (Figure 8B).

\section{DISCUSSION}

We found that undifferentiated human ES cells express AhR and Arnt, and have the capacity for endogenous TCDD-mediated AhR/Arnt signal transduction resulting in CYP1A1 induction. However, no observable morphological effects were detected in undifferentiated human ES cells following exposure to TCDD. When cells were grown in an environment permissive of spontaneous differentiation, colonies in TCDD-treated human ES cell cultures retained an undifferentiated morphology. In contrast, time-matched control cultures displayed signs of differentiation including longer, fibroblast-like cell morphology and high levels of phase-dense material accumulation. Relative to control human ES cells, TCDD-treated cells possessed higher protein levels of the pluripotency marker Oct4, while maintaining low transcript levels of common markers of meso- and endodermal differentiation. Furthermore, markers of EMT were expressed at lower levels in TCDDtreated cells in comparison with the vehicle controls, suggesting that the observed TCDDinduced inhibition in cellular differentiation may act by inhibiting an EMT-like mechanism present in early human ES cell differentiation. Together, these results suggest that there is a window during early embryogenesis in which TCDD may have a greater impact on differentiating cell populations, and may ultimately affect cell fate decisions.

Model systems using human ES cells represent a novel approach to study early human developmental toxicity at timepoints that have been historically impossible to investigate. Because of the novelty of the field of stem cell toxicology, only a few studies have been published demonstrating the role of AhR in human ES cell differentiation [21, 22]. In 2004, Ginis et al. reported that ARNT was not expressed in the H1, H7 and H9 lineages [22], in contrast to our findings. This discrepancy may be attributed to very low expression levels of $A H R$ or $A R N T$ which were detectable using qPCR, but may not have been detected by the less sensitive RT-PCR methodology employed by 
Ginis et al. In our study, expression of $A H R$ and $A R N T$ was greater in both 8-day cultures, as well as 15-day spontaneously differentiating cultures compared to earlier timepoints. This observation is consistent with reports of an increase in expression of both $A H R$ and ARNT in normal developmental processes in rodents [23, 24]. Recently, activation of the AhR pathway by TCDD has been shown in mouse ES cells by upregulating cytochrome P450 transcription [25-27].

The induction of CYP1A1 following both TCDD treatment and single-cell suspension culture further demonstrated the presence of AhR and Arnt, since both proteins are required for pathway activation and subsequent transcriptional upregulation of CYP1A1. Furthermore, transient induction of CYP1A1 following single-cell suspension suggests that cell-cell adhesion and interaction contribute to AhR pathway activation in human ES cells. Although the mechanism of suspension-mediated CYP1A1 induction is unknown, it has been speculated that loss of cell-cell adhesion may either increase the concentration of an unknown AhR agonist or spatially encourage the translocation of the AhR-Arnt heterodimer into the nucleus (reviewed in [28]). It is reasonable to speculate that the changing cell-cell adhesion dynamics present in human ES cells undergoing spontaneous differentiation may allow for greater translocation of TCDD-bound AhR to the nucleus, resulting in greater changes in gene expression compared to undifferentiated human ES cells.

As neuroectodermal markers can be detected in the absence of serum and other differentiationinducing reagents, formation of the ectodermal layer is believed to be the "default" pathway in ES cell and embryonic development (reviewed in [29]). In mouse ES cells, signaling events that promote primitive streak formation and subsequent meso- and endodermal germ layers include the Wnt, Activin and BMP pathways [29]. The activation of these pathways concurrently inhibits formation of ectodermal layers. Furthermore, suppression of BMP signaling, combined with addition of bFGF has been shown to be crucial for maintaining an undifferentiated population of human ES cells in culture [30]. Our results demonstrate strong inhibition of meso- and endodermal markers in human ES cells exposed to
TCDD, while expression of ectodermal markers remains unaffected. Our findings suggest that TCDD may have an extremely early effect on human development affecting genes known to play a role in cells during primitive streak formation. In support of this notion, lower transcript levels of the mesodermal marker BMP4 were observed in the presence of TCDD. Whether TCDD directly inhibits expression of differentiation markers, or if a TCDD-induced reduction in BMP4 indirectly contributes to lower expression of mesodermal and endodermal markers remains to be explored.

The low levels of mesodermal markers observed in TCDD-treated cultures lead us to investigate the effects of TCDD on morphological and molecular markers of EMT in human ES cells. EMT is a developmental process that has been demonstrated in vitro using both human and nonhuman primate ES cells [17-20, 31-33]. The protein localization patterns we observed in colonies of differentiating control human ES cells are consistent with models of EMT described in the literature in which the epithelial marker, E-cadherin, was observed in the center of cell colonies. Similar to previous reports we found that mesenchymal markers such as $\mathrm{N}$-cadherin and vimentin were located exclusively at the colony's periphery in differentiating human ES cells $[17,18]$. Continuous treatment of spontaneously differentiating human ES cell cultures with TCDD appeared to inhibit progression of EMT as evidenced by a reduction of $\mathrm{N}$-cadherin protein as well as absence of $\mathrm{N}$-cadherin localization in cells at the periphery of colonies. Attenuated Wnt1 and Wnt3 mRNA levels, coupled with low and in some cases blocked steady state mRNA levels for pro-EMT markers (snail, slug, N-cadherin, vimentin, MMP-2, and MMP-9) were observed in TCDD-treated human ES cultures. Together, these findings suggest that TCDD inhibits EMT in human ES cells.

Similar to the impact of TCDD upon cellular differentiation, activation of the AhR pathway has been shown to both enhance and inhibit EMT depending on species, cell and tissue type. In 2012, Zucchini-Pascal et al. found that treatment with TCDD and other organochlorines induced EMT in primary hepatocytes, as evidenced by mesenchymal morphology coupled with changes 
in EMT protein expression [34]. Other indirect evidence such as increased expression of MMP transcripts suggests TCDD-induced enhancement of EMT in human keratinocytes and lung carcinoma cell culture [35, 36]. Similarly, exposure to TCDD in vivo resulted in increased expression of ECM remodeling gene transcripts in mouse hearts; however no direct link to EMT was established [37]. In contrast to the committed adult cell types used in the investigations described above, our work with spontaneously differentiating human ES cells represents a highly plastic cell type, which may account for robust differences in EMT marker expression following TCDD exposure.

Consistent with our findings using human ES cells, TCDD has also been shown to inhibit EMT, as demonstrated through the well-accepted model of TCDD-induced cleft palate in mice and humans [10, 38-41]. In this pathology, TCDD is thought to inhibit epithelial migration and subsequently block fusion of the palatal shelves [42]. Additionally, transcriptional analysis of zebrafish exposed to TCDD shows impaired tailfin regeneration upon injury coupled with decreased expression of several matrix remodeling markers, developmental markers, as well as the mesenchymal marker, vimentin [43]. Most recently, it has been shown that activation of AhR inhibits EMT in epithelial cell culture, while inhibition of AhR leads to expression of pro-EMT markers [44], further demonstrating the impact of TCDD upon the differentiation progression of cell populations. Our investigation contributes new knowledge to this collection of TCDD-induced developmental effects by demonstrating that TCDD inhibits EMT in a population of differentiating human ES cells.

\section{CONCLUSION}

In summary, we have demonstrated the presence and functionality of $A H R$ and $A R N T$ in undifferentiated, pluripotent human ES cells. Furthermore we have shown that TCDD forestalled spontaneous differentiation in human ES cells while maintaining markers of pluripotency. We conclude that TCDD affects human ES cells during the early stages of germ layer formation. This conclusion is supported by the TCDDmediated attenuation of both morphological events (EMT) and molecular markers typically observed during ES cell differentiation. It is unknown if the effects of TCDD on human ES cell pluripotency and differentiation are direct or indirect. We provide the first evidence that EMT may be a target for early developmental effects of TCDD in human ES cells. Additional studies are needed to define specific pathways which contribute to the biological responses we observed in these pluripotent human cells. We speculate that the inhibition of human ES cellular differentiation observed in our studies may represent a protectionbased mechanism to prevent xenobiologicalinduced harm to the human fetus during very early stages of development.

\section{ACKNOWLEDGEMENTS}

We thank Peggy J. Rooney, Cathy A. Rasmussen, and Christina L. Thomas-Virnig for editorial assistance with this manuscript. This work was supported by National Institutes of Health grant R01 HL074284.

\section{CONFLICT OF INTEREST STATEMENT}

None to declare.

\section{REFERENCES}

1. Rolletschek, A., Blyszczuk, P. and Wobus, A. M. 2004, Toxicol. Lett., 149, 361.

2. Thomson, J. A., Itskovitz-Eldor, J., Shapiro, S. S., Waknitz, M. A., Swiergiel, J. J., Marshall, V. S. and Jones, J. M. 1998, Science, 282, 1145.

3. Wobus, A. M. and Loser, P. 2011, Arch. Toxicol., 85, 79.

4. van Dartel, D. A. M. and Piersma, A. H. 2011, Reproductive Toxicology, 32, 235.

5. Laustriat, D., Gide, J. and Peschanski, M. 2010, Biochem. Soc. Trans., 38, 1051.

6. Bock, K. W. and Kohle, C. 2006, Biochem. Pharmacol., 72, 393.

7. Bock, K. W. 2013, Biol. Chem., 394, 729.

8. Panteleyev, A. A. and Bickers, D. R. 2006, Exp. Dermatol., 15, 705.

9. Abbott, B. D. and Birnbaum, L. S. 1991, Teratology, 43, 119.

10. Moriguchi, T., Motohashi, H., Hosoya, T., Nakajima, O., Takahashi, S., Ohsako, S., Aoki, Y., Nishimura, N., Tohyama, C., Fujii-Kuriyama, Y. and Yamamoto, M. 2003, Proc. Natl. Acad. Sci. USA, 100, 5652. 
11. Whitsett, Jr. T. G., Kalia, V., Eltoum, I. and Lamartiniere, C. A. 2008, The Open Toxicology Journal, 2, 13.

12. Gray, L. E. Jr. and Ostby, J. S. 1995, Toxicol. Appl. Pharmacol., 133, 285.

13. Flaws, J. A., Sommer, R. J., Silbergeld, E. K., Peterson, R. E. and Hirshfield, A. N. 1997, Toxicol. Appl. Pharmacol., 147, 351.

14. Rasmussen, C. 2009, dissertation.

15. Sadek, C. M. and Allen-Hoffmann, B. L. 1994, J. Biol. Chem., 269, 16067.

16. Loertscher, J. A., Lin, T. M., Peterson, R. E. and Allen-Hoffmann, B. L. 2002, Toxicol. Sci., 68, 465.

17. Eastham, A. M., Spencer, H., Soncin, F., Ritson, S., Merry, C. L., Stern, P. L. and Ward, C. M. 2007, Cancer Res., 67, 11254.

18. Ullmann, U., In’t Veld, P., Gilles, C., Sermon, K., De Rycke, M., Van de Velde, H., Van Steirteghem, A. and Liebaers, I. 2007, Mol. Hum. Reprod., 13, 21.

19. Chan, D. N., Azghadi, S. F., Feng, J. and Lowry, W. E. 2012, PLoS One, 7, e50432.

20. Evseenko, D., Zhu, Y., Schenke-Layland, K., Kuo, J., Latour, B., Ge, S., Scholes, J., Dravid, G., Li, X., MacLellan, W. R. and Crooks, G. M. 2010, Proceedings of the National Academy of Sciences, 107, 13742.

21. Kee, K., Flores, M., Cedars, M. I. and Reijo Pera, R. A. 2010, Toxicol. Sci., 117, 218.

22. Ginis, I., Luo, Y., Miura, T., Thies, S., Brandenberger, R., Gerecht-Nir, S., Amit, M., Hoke, A., Carpenter, M. K., ItskovitzEldor, J. and Rao, M. S. 2004, Dev. Biol., 269, 360.

23. Abbott, B. D., Birnbaum, L. S. and Perdew, G. H. 1995, Dev. Dyn., 204, 133.

24. Abbott, B. D., Held, G. A., Wood, C. R., Buckalew, A. R., Brown, J. G. and Schmid, J. 1999, Toxicol. Sci., 47, 62.

25. Neri, T., Merico, V., Garagna, S., Redi, C. A. and Zuccotti, M. 2008, Biochim. Biophys. Acta, 20, 20.

26. Neri, T., Merico, V., Fiordaliso, F., Salio, M., Rebuzzini, P., Sacchi, L., Bellazzi, R., Redi, C. A., Zuccotti, M. and Garagna, S. 2011, Toxicol. Lett., 202, 226.
27. Wang, Y., Fan, Y. and Puga, A. 2010, Toxicol. Sci., 3, 3.

28. Barouki, R., Coumoul, X. and FernandezSalguero, P. M. 2007, FEBS Lett., 581, 3608.

29. Murry, C. E. and Keller, G. 2008, Cell, 132, 661.

30. Xu, R. H., Peck, R. M., Li, D. S., Feng, X., Ludwig, T. and Thomson, J. A. 2005, Nat. Methods, 2, 185.

31. Ullmann, U., Gilles, C., De Rycke, M., Van de Velde, H., Sermon, K. and Liebaers, I. 2008, Mol. Hum. Reprod., 14, 169.

32. Denker, H.-W., Behr, R., Heneweer, C., Viebahn, C. and Thie, M. 2007, Cells Tissues Organs, 185, 48.

33. Behr, R., Heneweer, C., Viebahn, C., Denker, H. W. and Thie, M. 2005, Stem Cells, 23, 805.

34. Zucchini-Pascal, N., Peyre, L., de Sousa, G. and Rahmani, R. 2012, Food Chem. Toxicol., 50, 3963.

35. Martinez, J. M., Afshari, C. A., Bushel, P. R., Masuda, A., Takahashi, T. and Walker, N. J. 2002, Toxicol. Sci., 69, 409.

36. Murphy, K. A., Villano, C. M., Dorn, R. and White, L. A. 2004, J. Biol. Chem., 279, 25284.

37. Aragon, A. C., Kopf, P. G., Campen, M. J., Huwe, J. K. and Walker, M. K. 2008, Toxicol. Sci., 101, 321.

38. Abbott, B. D. and Birnbaum, L. S. 1989, Toxicol. Appl. Pharmacol., 99, 287.

39. Thomae, T. L., Stevens, E. A. and Bradfield, C. A. 2005, J. Biol. Chem., 280, 12742.

40. Nogai, H., Rosowski, M., Grun, J., Rietz, A., Debus, N., Schmidt, G., Lauster, C., Janitz, M., Vortkamp, A. and Lauster, R. 2008, Differentiation, 76, 404.

41. Yu, W., Ruest, L. B. and Svoboda, K. K. 2009, Exp. Biol. Med. (Maywood), 234, 483.

42. Abbott, B. D. and Birnbaum, L. S. 1990, Toxicology and Applied Pharmacology, 106, 418.

43. Andreasen, E. A., Mathew, L. K. and Tanguay, R. L. 2006, Toxicol. Sci., 92, 254.

44. Rico-Leo, E. M., Alvarez-Barrientos, A. and Fernandez-Salguero, P. M. 2013, J. Biol. Chem., 288, 7841. 